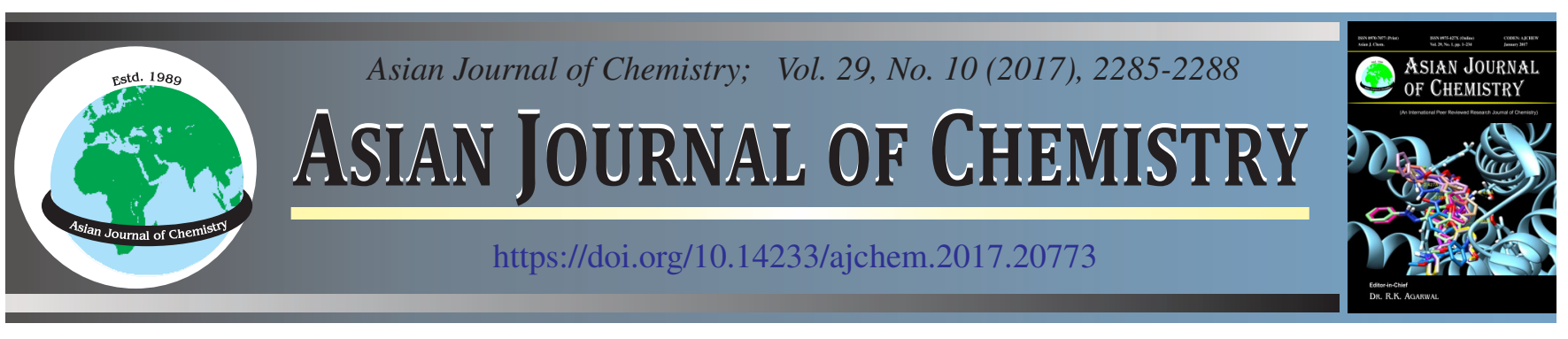

\title{
Improvement of Thermal Properties of Poly(vinyl chloride) Using Chemical Blending Assisted Ultrasonic Technique
}

\author{
AzZa Al-GHAmdi
}

Chemistry Department, College of Science, University of Dammam, Dammam, Saudi Arabia

Corresponding author: E-mail: azaalghamdi@uod.edu.sa

Received: 9 May 2017;

Accepted: 30 June 2017;

Published online: 31 August 2017;

AJC-18535

The thermal stabilization of poly(vinyl chloride) through blending techniques has been studied. Poly(vinyl chloride) was blended with modified polymer (cellulose acetate-diallyl amine) in different compositions to improve the thermal stability of poly(vinyl chloride). The thermal stability and morphology of the blend films were characterized by scanning electron microscope (SEM) and thermogravimetry.

The results revealed that the presence of modified cellulose acetate improved the thermal stability of poly(vinyl chloride). This was attributed to the thermal stable diallylamine moieties among the cellulose acetate chains. The addition of traces of maleimide derivatives to poly(vinyl chloride) prior to the blend process led to an extra thermal stability of the blend film as shown from the values of the initial decomposition temperature $\left(\mathrm{T}_{\mathrm{o}}\right)$ measured by thermogravimetry.

Keywords: Poly(vinyl chloride), Ultrasonic waves, Thermal properties.

ᄂ - - - - - - - - - - - - - - - - - - - - - - - - - -

\section{INTRODUCTION}

Poly(vinyl chloride) (PVC) is widely used in plastic applications due to low cost and ease handling process. The thermal in-stability during molding and the photo-degradation during day use are the major problems facing the industrial benefit of this polymer. Thus, to use PVC to industrial applications, it should be thermally stabilized to support the polymer during molding process and against the photo-degradation [1-4].

The heat instability is definitely due the structural defects formed during polymerization reaction. These defects are mainly, allylic or tertiary labile chlorine atoms, which cause the formation of polyenes after losing $\mathrm{HCl}$ [5].

The use of stabilizers is either for absorbing the evolved gas or displacing the labile sites. The commercially used stabilizers are usually containing heavy metals which are environmentally rejected. To overcome the accumulation of these heavy metals, organic based stabilizers were used as additives [6,7] or modifying agents $[8,9]$. Moreover, polymer blend technique is also considered as effective and commercial method to stabilize such a polymer [10-13], these stabilizers are environmentally accepted $[14,15]$. The aim of this work is to stabilize poly(vinyl chloride) by blending it with suitable biodegradable polymers through their amorphous regions to improve its thermal stability to enable the adjustment of poly(vinyl chloride) for plastics applications [16,17].

\section{EXPERIMENTAL}

Poly(vinyl chloride) (PVC) (suspension), K value 67, from National (Plastic Company, Sabic), (Kingdom of Saudi Arabia). Cellulose acetate (CA) was purchased from (Aldrich) with a degree of polymerization and degree of substitution of 160 and 2.4 , respectively.

Aniline was purified by vacuum distillation, 4-chloro aniline (Acros), 4-bromo aniline (Acros) and maleic anhydride were recrystallized from acetone. Tetrahydrofuran was obtained from (Prolabo) (used after distillation in the presence of $\mathrm{KOH}$ pellets). Other reagents and solvents were of analytical grades and used as received.

Thermal characterization: Thermogravimetric analysis experiments were examined using simultaneous thermal gravimetric analyzer a Perkin Elmer thermalgravimetric analyzer (TGA) under nitrogen atmosphere and the heating rate is $10{ }^{\circ} \mathrm{C} / \mathrm{min}$.

Morphological surface analysis: Poly(vinyl chloride) morphology in presence of cellulose acetate, modified cellulose acetate and additives was examined by emission scanning electron microscopy (ESEM) using a (FEG-SEM/EDS)or LEO 440ZEISS/LEICA model.

Methods: N-Phenyl maleimide and their derivatives synthesized in two steps using aniline derivatives and maleic anhydride. 
Preparation of N-phenyl maleimide: Aniline $(0.1 \mathrm{~mol})$ and maleic anhydride $(0.1 \mathrm{~mol})$ were dissolved in $50 \mathrm{~mL}$ chloroform. The reaction was stirred for $3 \mathrm{~h}$ at room temperature $\left(20-25^{\circ} \mathrm{C}\right)$. The solid maleaimic acid derivatives were filtered and dried.

The maleamic acid was allowed to cyclodehydrated by using acetic anhydride and fused sodium acetate. The solution was stirred for $0.5 \mathrm{~h}$ at $80{ }^{\circ} \mathrm{C}$. The prepared produced is precipitated in ice, filtered and washed by water. The product was then crystallized by ethanol [6] (Fig. 1).

Preparation of modified cellulose acetate: Cellulose acetate modified by diallylamine was prepared by adding diallylamine to a solution of cellulose acetate in cyclohexanone according to the method described by Abdel-Naby et al. [18]. The modification reaction occurs in ultrasonic bath of power $1050 \mathrm{~W}$ for $2 \mathrm{~h}$ at $60^{\circ} \mathrm{C}$.

Preparation of blend film: The PVC compounding (PVC $+0.02 \% \mathrm{~N}$-phenyl maleimide derivatives) sample solution is blended with cellulose acetate or modified cellulose acetate solution in an ultrasonic bath for certain interval of time till homo-geneous solution is obtained. The casting technique is used to obtain the blend film after the evaporation of the solvent at room temperature.

\section{RESULTS AND DISCUSSION}

Effect of ultrasonic waves on homogeneity of the blend films: Ultrasonic waves are known to possess the ability to initiate chemical reaction in solution [19] as they provide fast particles motion. They enhanced the interaction between the two polymers and thus improved the homogeneity of the film (Fig. 2). The morphology of (70:30) [PVC/(CA-DAA)] blend film showed very limited homogeneity feature (b). However, the use of ultrasonic bath during the blending process improved the homogeneity of the blend film as seen from the morphology feature (c).
Thermogravimetry (TG): Another example for the assistance of ultrasonic waves is their effect on the thermal stability of the blend film. It was clearly found that the ultrasonic waves assisted the homogeneity of the blend film since the thermal stability of the blend films prepared in ultrasonic bath exhibited higher thermal stability than that prepared without ultrasonic waves. Fig. 3 and Table- 1 showed $\mathrm{T}_{\mathrm{o}}$ value (initial decomposition temperature) of the blend films. The results revealed that the films prepared using ultrasonic waves which showed homogeneous feature exhibited higher value $\left(\mathrm{T}_{\mathrm{o}}=217^{\circ} \mathrm{C}\right)$ as compared to those showing limited homogeneity and exhibiting lower value $\left(\mathrm{T}_{\mathrm{o}}=200{ }^{\circ} \mathrm{C}\right)$. This might be attributed to the help of ultrasonic waves on providing better distribution of both polymers through their amorphous regions.

\begin{tabular}{|c|c|c|c|}
\hline \multicolumn{4}{|c|}{$\begin{array}{c}\text { TABLE-1 } \\
\text { EFFECT OF ULTRASONIC WAVES ON THE THERMAL } \\
\text { STABILITY BLEND FILM (70:30) (PVC:CA) IN PRESENCE } \\
\text { AND ABSENCE OF MODIFIED CELLULOSE ACETATE (CA) }\end{array}$} \\
\hline Blend films & $\begin{array}{c}\text { Ultrasonic } \\
\text { waves exposure }\end{array}$ & $\begin{array}{c}\mathrm{T}_{\mathrm{o}} \\
\left({ }^{\circ} \mathrm{C}\right)\end{array}$ & $\begin{array}{l}\text { Total weight loss } \\
(\%) \text { at } 500^{\circ} \mathrm{C}\end{array}$ \\
\hline Poly(vinyl chloride) & 0 & 180 & 88.7 \\
\hline Cellulose acetate & 0 & 260 & 89.3 \\
\hline [PVC-CA] & 0 & 200 & 84.4 \\
\hline [PVC-CA] & 2 & 217 & 83.7 \\
\hline [PVC-(CA/DAA)] & 2 & 240 & 82.7 \\
\hline
\end{tabular}

Comparing the thermogravimetry of (PVC-CA), it was found that $T_{0}$ value increased with the using modified cellulose acetate (CA/DAA) as compared with free cellulose acetate (Fig. 3). This is attributed to the increase in the crystalline regions provided by modified cellulose acetate polymeric matrix [18].

The [PVC-(CA/DAA)] (70:30) blend film exhibited the highest thermal stability as shown from the highest value of $\left(\mathrm{T}_{\mathrm{o}}=240{ }^{\circ} \mathrm{C}\right)$.

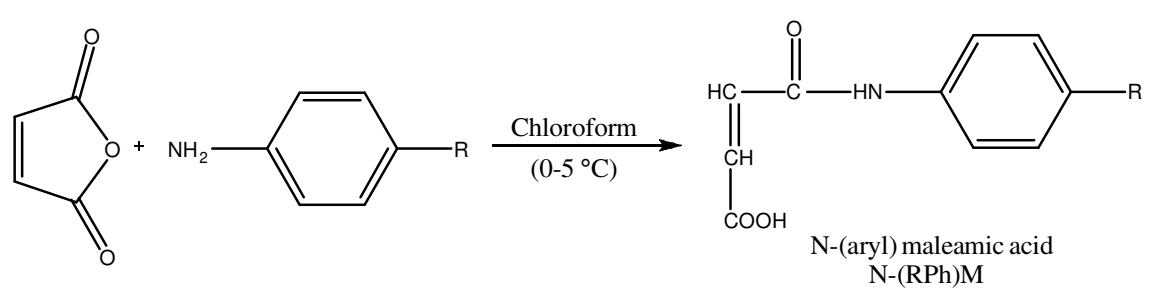

Fig. 1. Preparation of N-phenyl malemide [Ref. 6]

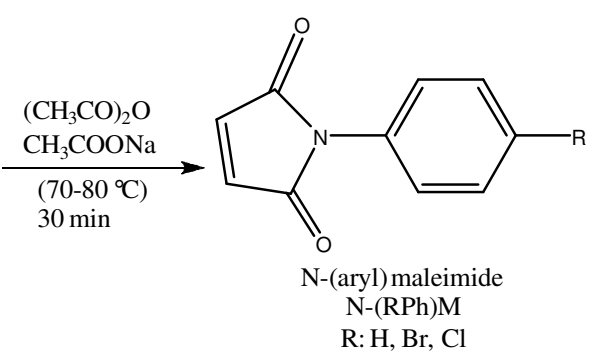

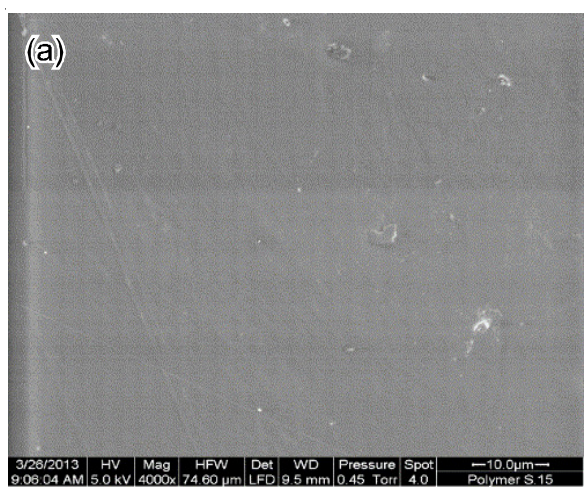
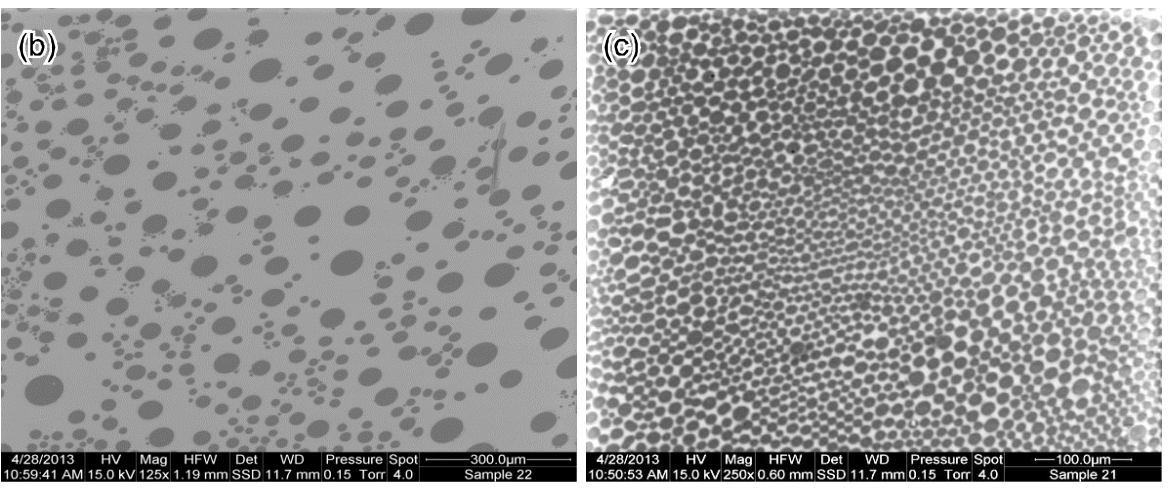

Fig. 2. ESEM of (a) PVC, (b) (70:30) [PVC-(CA/DAA)] prepared in water bath and (c) (70:30) [PVC-(CA/DAA)] Prepared in ultrasonic bath for $2 \mathrm{~h}$ 
However, the PVC compounding is known to contain no more than $20 \%$ additives to keep the plasticity of the film unaffected $[17,18]$. In a trial to give the blend film extra thermal stability, the PVC is mixed with traces of $\mathrm{N}$-phenyl maleimide prior to blend it with cellulose acetate.

The thermal stability of the thermogravimetry blend films with (PVC/CA) composition (70:30) in absence and presence of traces of N-phenyl maleimide derivatives was shown in Fig. 4. The blend films in presence of halophenylmaleimide derivatives exhibited high $\mathrm{T}_{\mathrm{o}}$ values as compared to that in absence maleimide derivatives or even in presence of unsubstituted N-phenyl maleimide. This might be attributed to secondary forces provided from the interaction of halo groups and oxygen atom of the hydroxyl group of glucopyranose ring of cellulose acetate (Fig. 5).



Fig. 3. Thermogravimetry (TG) of (a) CA, (b) PVC, (d) (70:30) (PVC$\mathrm{CA})$ and $(\mathrm{e})(70: 30)$ [PVC-(CA/DAA)] blended with ultrasonic waves for $2 \mathrm{~h}$, (c) (PVC-CA) blended without ultrasonic waves

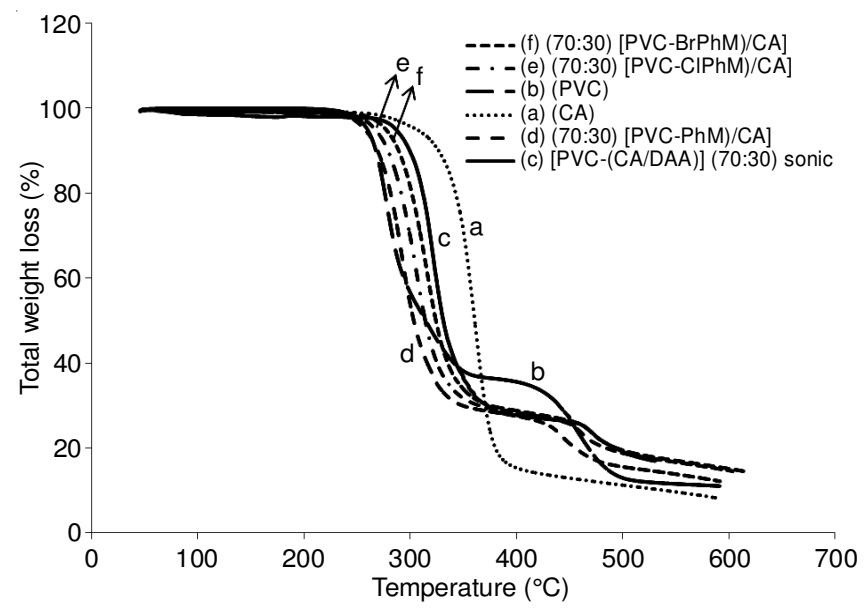

Fig. 4. Thermogravimetry (TG) of (a) (CA), (b) (PVC), (c) (70:30) [PVC(CA/DAA)], (d) (70:30) [(PVC-PhM)/CA], (e) (70:30) [(PVC$\mathrm{ClPhM} / \mathrm{CA}]$

Fig. 4 and Table-2 revealed that the presence of modified cellulose acetate gave the extra thermal stability of the film as shown from the high values of $\mathrm{T}_{\mathrm{o}}$ as compared to the blend film in presence of unmodified cellulose acetate and 4-bromomalimide $\left(\mathrm{T}_{\mathrm{o}}=230^{\circ} \mathrm{C}\right)$.

Morphological characterization: The morphology of the surface of PVC blend films in presence of cellulose acetate and additives are shown in Fig. 6.

\section{Conclusion}

The PVC blend polymers with cellulose acetate, modified cellulose acetate and additives have been prepared to improve the thermal stability of PVC. The thermal stability of blend


(a)<smiles>[X]c1ccc(N2C(=O)C=CC2=O)cc1</smiles><smiles>[X]c1ccc(N(C(=O)Cl)C(=O)C(C)Cl)cc1</smiles><smiles>[X]c1ccc(N(C(=O)Cl)C(=O)C(Cl)C(C=CCC)C(C)C)cc1</smiles>



(b)

(a)

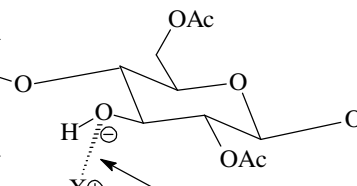

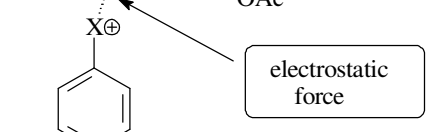

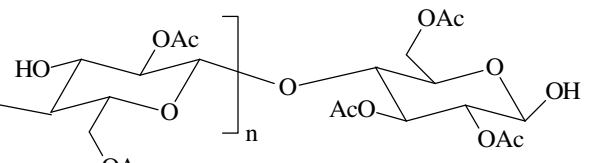<smiles>CC/C=C/C(CC)C1C(=O)N(c2ccc([123I])cc2)C(=O)C1Cl</smiles>

Fig. 5. Effect of halogen groups in enhancing both the incorporation of $\mathrm{N}$-(4-halo) maleimide molecules into the PVC chains and the compatibility of PVC with cellulose acetate chains 

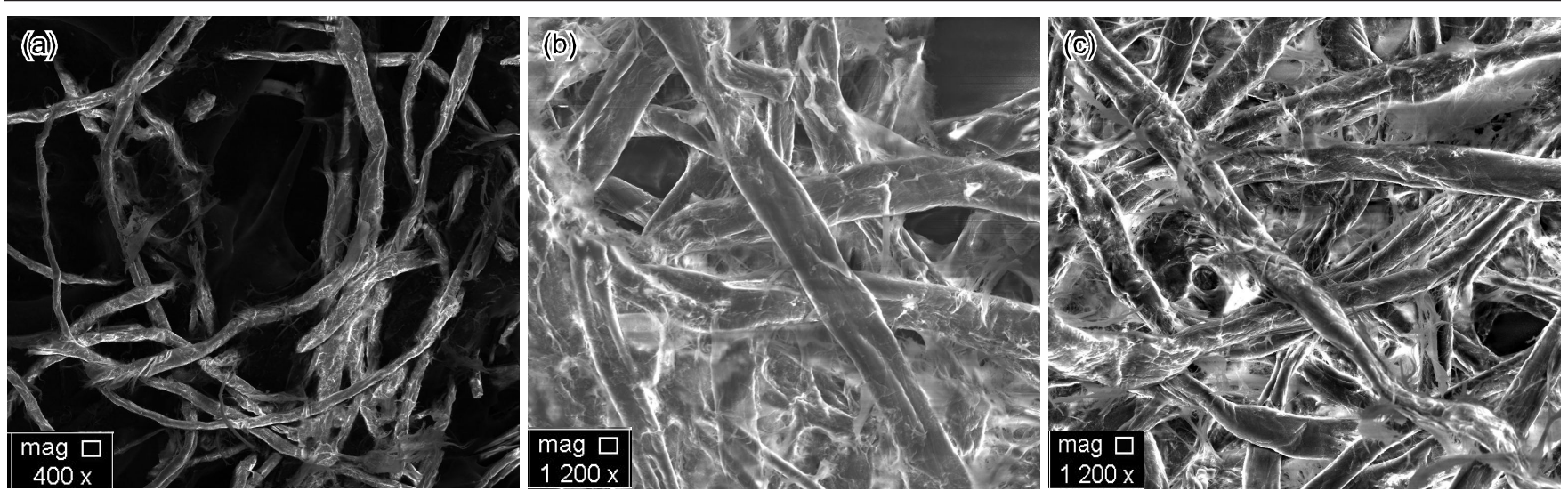

Fig. 6. ESEM morphology of polymeric films (a) [PVC-PhM/CA], (b) [PVC-4-ClPhM/CA] and (c) [PVC-4-BrPhM/CA]

TABLE-2

THERMAL ANALYSIS OF VARIOUS BLEND FILM (70:30) (PVC:CA) IN PRESENCE AND ABSENCE OF TRACES OF XPhM DERIVATIVES PREPARED IN ULTRASONIC BATH FOR $2 \mathrm{~h}$

\begin{tabular}{lcc}
\hline \multicolumn{1}{c}{ Blend films } & $\mathrm{T}_{\mathrm{o}}\left({ }^{\circ} \mathrm{C}\right)$ & Total weight loss $(\%)$ at $500{ }^{\circ} \mathrm{C}$ \\
\hline PVC & 180 & 88.7 \\
CA & 260 & 89.3 \\
[PVC-CA] & 217 & 90.0 \\
[PVC-PhM/CA] & 213 & 87.7 \\
[PVC-ClPhM/CA] & 220 & 85.4 \\
{$[$ PVC-BrPhM/CA] } & 230 & 85.4 \\
\hline
\end{tabular}

films increases with using (CA/DAA) content in the blend film rather than using additives as shown from the higher value of $\mathrm{T}_{\mathrm{o}}$ for the former as compared to the unmodified cellulose acetate. The results also revealed that [PVC-(CA/DAA)] blend film possessed higher $\mathrm{T}_{\mathrm{o}}$ value than that of PVC-phenyl maleimide derivatives.

\section{ACKNOWLEDGEMENTS}

This project no. (2015102) was funded By Deanship of Scientific Research at University of Dammam.

\section{REFERENCES}

1. N. Mohamed and M. Sabaa, Eur. Polym. J., 35, 1731 (1999); https://doi.org/10.1016/S0014-3057(98)00257-2.

2. A. Abdel-Naby and M. El-Hefnawy, Polym. Test., 22, 25 (2003); https://doi.org/10.1016/S0142-9418(02)00044-2.

3. A. Abdel-Naby, Am. J. Appl. Sci., 8, 675 (2011); https://doi.org/10.3844/ajassp.2011.675.680.

4. A. Abdel-Naby and A. Al-Ghamdi, Int. J. Biol. Macromol., 70, 124 (2014); https://doi.org/10.1016/j.ijbiomac.2014.06.033.

5. W.H. Starnes, J.A. Wallach and H. Yao, Macromolecules, 30, 676 (1997); https://doi.org/10.1021/ma9520252.
6. N.A. Mohamed and W.M. Al-Magribi, Polym. Degrad. Stab., 82, 421 (2003);

https://doi.org/10.1016/S0141-3910(03)00194-0.

7. A. Abdel-Naby and A. Al Dossary, J. Vinyl Addit. Technol., 14, 175 (2008); https://doi.org/10.1002/vnl.20165.

8. A. Torikai and H. Hasegawa, Polym. Degrad. Stabil., 63, 441 (1999); https://doi.org/10.1016/S0141-3910(98)00125-6.

9. C. Banzato, V. Massimo, C. Fiorucci, B. Vigna and S. Papale, Am. J. Environ. Sci., 7, 1 (2011); https://doi.org/10.3844/ajessp.2011.1.14.

10. A. Yassin, M. Sabaa and A. Abdel-Naby, Polym. Degrad. Stab., 31, 189 (1991); https://doi.org/10.1016/0141-3910(91)90074-2.

11. A. Abdel-Naby and A. Youssef, Polym. Int., 46, 336 (1998); https://doi.org/10.1002/(SICI)1097-0126(199808)46:4<336::AIDPI34>3.0.CO;2-B.

12. A.S. Andel-Naby, J. Vinyl Addit. Technol., 5, 159 (1999); https://doi.org/10.1002/vnl.10326.

13. A. Abdel-Naby, R. Al-Ghamdi and A. Al-Ghamdi, J. Addit. Technol., 16, 15 (2010); https://doi.org/10.1002/vnl.20225.

14. Z. Vymazal, K. Volka, M.W. Sabaa and Z. Vymazalová, Eur. Polym. J., 19, 63 (1983); https://doi.org/10.1016/0014-3057(83)90103-9.

15. M.K. Naqvi, P.A. Unnikrishnan, Y.N. Sharma and I.S. Bhardwaj, Eur. Polym. J., 20, 95 (1984); https://doi.org/10.1016/0014-3057(84)90231-3.

16. A. Abdel-Naby, J. Vinyl Addit. Technol., 7, 244 (2001); https://doi.org/10.1002/vnl.10296.

17. A. Abdel-Naby and A. Al Dossary, J. Vinyl Addit. Technol., 14, 167 (2008); https://doi.org/10.1002/vnl.20159.

18. A. Abdel-Naby and A. Al-Ghamdi, Int. J. Curr. Microbiol. Appl. Sci., 3, 10 (2014)

19. G. Zaikov, Chemistry of Polysaccharides, Taylor \& Francis Group, USA (2005).

20. M.W. Sabaa, M.G. Mikheal, A.A. Yassin and M.Z. Elsabee, Angew. Makromol. Chem., 139, 95 (1986); https://doi.org/10.1002/apmc.1986.051390110. 